\title{
Empathie, biais de mentalisation, comportements pro-sociaux et troubles de comportement chez les enfants d'âge scolaire
}

\author{
Émilie Girard, Miguel M. Terradas et Célia Matte-Gagné
}

Volume 3, novembre 2014

URI : https://id.erudit.org/iderudit/1028013ar

DOI : https://doi.org/10.7202/1028013ar

Aller au sommaire du numéro

Éditeur(s)

Université Laurentienne

ISSN

1920-6275 (imprimé)

1929-8544 (numérique)

Découvrir la revue

Citer cet article

Girard, É., Terradas, M. M. \& Matte-Gagné, C. (2014). Empathie, biais de mentalisation, comportements pro-sociaux et troubles de comportement chez les enfants d'âge scolaire. Enfance en difficulté, 3, 77-105.

https://doi.org/10.7202/1028013ar
Résumé de l'article

La présente étude propose d'examiner les liens entre la capacité d'empathie, les comportements pro-sociaux, les biais de mentalisation et les troubles de comportement (TC) chez les garçons âgés de 7 à 12 ans. Pour ce faire, 51 garçons présentant divers niveaux de TC et leurs parents ont participé à la recherche. Les enfants ont rempli des questionnaires autoadministrés concernant l'empathie et les biais de mentalisation. Pour leur part, les parents ont rempli des questionnaires relatifs à l'empathie, aux comportements pro-sociaux et aux TC manifestés par leurs enfants. Les résultats démontrent que les biais de mentalisation négatifs et les comportements pro-sociaux prédisent $24,5 \%$ de la variance des TC. La capacité d'empathie quant à elle serait une variable médiatrice entre les comportements pro-sociaux et la présence des TC. Les résultats de la recherche permettront d'ajuster les programmes de prévention des TC et les interventions offertes aux enfants d'âge scolaire présentant ces troubles. 
www.laurentienne.ca

\title{
Empathie, biais de mentalisation, comportements pro-sociaux et troubles de comportement chez les enfants d'âge scolaire
}

\author{
Émilie Girard, Miguel M. Terradas et Célia Matte-Gagné \\ Université de Sherbrooke
}

Les troubles de comportement (TC) constituent une problématique de plus en plus importante dans le système d'éducation québécois (Massé, Desbiens et Lanaris, 2006). La proportion de jeunes qui présentent ce type de difficultés tend à augmenter d'année en année (Fortin, Marcotte, Royer et Potvin, 2000). Il a été également observé que les garçons sont surreprésentés au sein de cette problématique par rapport aux filles (Ministère de l'Éducation, du Loisir et du Sport; MÉLS, 2010). La définition des enfants présentant des TC en milieu scolaire réfère à des déficits marqués au niveau de l'adaptation qui se manifestent par des difficultés d'interaction avec leur environnement social, scolaire ou familial (Tremblay et Royer, 1992). Les comportements problématiques de ces enfants sont majoritairement d'ordre surréactif et doivent être assez présents pour nuire au développement de l'enfant ou à celui d'autrui. Les enfants présentant des comportements surréactifs sont généralement hyperactifs, agressifs, désobéissants, et peuvent développer des troubles des conduites (Tremblay et Royer, 1992). On observe également chez ces enfants la présence de déficits marqués sur le plan des fonctions exécutives (Barkley, 1997, 2006; Baving, Rellum, Laucht et Schmidt, 2006; Greene et Doyle, 1999; Harman, Rothbart et Posner, 1997), fonctions qui sont essentielles pour la régulation du comportement, des émotions, de la pensée et des fonctions cognitives (Chevalier, 2010). Ainsi, il est important de mieux saisir les mécanismes 
sous-jacents aux TC afin d'être en mesure de mieux comprendre la chronicisation de ces troubles et de développer des moyens de prévention et d'intervention plus efficaces et ciblés.

Plusieurs recherches visent à connaître les facteurs émotionnels et comportementaux associés aux TC. Parmi ces facteurs, des études récentes démontrent que les difficultés quant à la capacité d'être empathique, d'adopter des comportements pro-sociaux et de prendre la perspective d'autrui sont fortement reliées à la présence de TC chez l'enfant (de Waal, 2008; Penner, Dovidio, Piliavin et Schroeder, 2005; Preston et de Waal, 2002). Blair, Peschardt, Budhani, Mitchell et Pine (2006) expliquent bien la distinction entre l'empathie et la capacité à prendre la perspective de l'autre. Ils définissent l'empathie comme une réponse affective plus appropriée à la situation de l'autre qu'à la sienne. Elle implique que l'on partage et que l'on éprouve en soi-même la qualité des sentiments d'autrui, sans nécessairement en ressentir la même intensité (Lafortune, 2008). L'empathie est une réponse émotionnelle concordante avec l'état affectif d'autrui qui se produit conséquemment à la capacité de l'observateur à prendre la perspective de l'autre. Cette capacité implique que l'individu doit être en mesure de réfléchir aux états mentaux d'autrui. Sont considérés des états mentaux les pensées, les désirs, les sentiments, les croyances et les intentions. En résumé, l'empathie serait une réponse émotionnelle qui arrive suite à la représentation des états mentaux d'une autre personne. Stetson (1998) renchérit d'ailleurs en rapportant que la capacité à prendre la perspective de l'autre est essentielle pour expliquer comment l'empathie peut engendrer des comportements pro-sociaux et diminuer ainsi les manifestations des TC.

Les comportements pro-sociaux sont des gestes volontaires qui ont pour objectif de faire bénéficier autrui de leurs conséquences positives, contribuant ainsi aux relations sociales (Alessandri, Caprara, Eisenberg et Steca, 2009; Batson, 1998; Eisenberg, Fabes et Spinrad, 2006; Penner et collab., 2005). Certains gestes comme le partage, les dons, la générosité, la compassion, ainsi que les comportements visant à prendre soin d'une autre personne, à la réconforter ou à l'aider, en font partie. Selon Stetson (1998), le fait de ressentir des émotions similaires à celles d'une autre personne pourrait motiver l'enfant à répondre d'une manière altruiste. Par contre, si l'enfant ne comprend pas le point de vue d'autrui, il peut s'avérer difficile pour lui de savoir quelle action pourrait être considérée comme étant aidante ou nuisible. Ainsi, les processus cognitifs (prendre la perspective de l'autre) et affectifs (empathie) sont 
tous deux essentiels à l'enfant afin qu'il puisse avoir une compréhension plus complète de l'expérience d'autrui. Cette compréhension se traduira par une motivation à émettre un comportement aidant qui, à son tour, diminuera la probabilité que l'enfant manifeste un comportement inadéquat (Stetson, 1998). Il est donc important de mieux comprendre la contribution de chacune des composantes de l'empathie (cognitive et affective) afin d'être en mesure de bien cerner la problématique des TC.

\section{La notion de mentalisation: une nouvelle avenue de compréhension des difficultés relationnelles des enfants présentant un TC}

Dans les écrits scientifiques, l'empathie et la mentalisation sont souvent décrites comme étant des concepts qui se chevauchent. Cependant, ce ne sont pas des synonymes (Bjorkqvist et Osterman, 2000). Ces concepts se complètent et sont nécessaires à une adaptation sociale optimale (Fonagy, 2003). Il est donc important de saisir l'apport des capacités d'empathie et de mentalisation pour être en mesure de bien comprendre ce qui entre en jeu lorsqu'un enfant décide ou non de poser un geste pro-social, ce geste pouvant contribuer à éviter les comportements externalisés inadéquats. Les études portant sur la mentalisation s'attardent également à l'empathie et à la capacité à prendre la perspective d'une autre personne, mais nomment ce dernier concept différemment. En effet, la définition de la composante cognitive de l'empathie, soit la capacité à prendre la perspective de l'autre, peut s'apparenter au concept de mentalisation d'autrui décrit par Fonagy et Target (2006). La capacité à prendre la perspective de l'autre réfère à la théorie de l'esprit et se définit comme la compréhension cognitive des sentiments et des comportements d'autrui (Baron-Cohen et Wheelwright, 2004). Pour sa part, la mentalisation concerne un acquis développemental essentiel qui permet à l'individu de percevoir et d'organiser ses propres comportements et ceux des autres sous forme d'états mentaux (Fonagy et Target, 2006). Cette fonction amène l'individu à interpréter, implicitement (de façon inconsciente, automatique, exprimée par des gestes) ou explicitement (de manière consciente, réflexive et mise en mots), ce qu'il ressent et ce qu'il vit en termes de pensées, de croyances, de sentiments, d'attentes, de désirs et d'intentions sous-jacentes (Allen, Fonagy et Bateman, 2008; Fonagy, Gergely, Jurist et Target, 2002). En résumé, la théorie de l'esprit réfère à la compréhension cognitive des sentiments et des comportements d'autrui. Étant une notion plus 
complexe, la mentalisation concerne les états mentaux d'autrui, mais également ceux de l'individu lui-même. Elle réfêre également à la capacité de l'individu à comprendre les influences mutuelles entre les états mentaux et les comportements chez soi et l'autre.

\section{Développement de la capacité de mentalisation}

La mentalisation se développe dès la naissance, en même temps que s'installe la relation entre la figure d'attachement et le nourrisson (Fonagy et Target, 2006; Midgley et Vrouva, 2012). La capacité de mentalisation d'un enfant se développerait mieux quand les membres de sa famille sont sensibles à ses états mentaux (Allen et collab., 2008). En effet, la fonction miroir (Winnicott, 1971), lorsqu'elle est exercée adéquatement par la figure d'attachement, permet de refléter à l'enfant son expérience interne de manière contingente, congruente, différenciée et marquée, ce qui la rend tolérable pour l'enfant. La contingence fait allusion à l'émission par la figure d'attachement d'une réponse dans un délai suffisamment rapproché du comportement ou de l'état de l'enfant afin qu'il comprenne que les deux évènements sont liés. Le reflet de la figure d'attachement est congruent lorsqu'il saisit la nature de la détresse de l'enfant. Il se différencie du comportement de ce dernier quand il est fondé sur l'état d'esprit de l'enfant et non sur celui de la figure d'attachement. Finalement, la réponse marquée de la figure d'attachement est une version intentionnellement exagérée de la réponse émotionnelle réaliste et normale qu'elle donnerait face à l'état interne que présente l'enfant (Fonagy et collab., 2002). Ce dernier a alors l'illusion que le traitement de son expérience interne s'est fait adéquatement et il peut alors l'intégrer. C'est de cette façon que l'enfant développe les premiers jalons de sa capacité de mentalisation et du self comme agent social (Fonagy, Steele, Steele, Moran et Higgitt, 1991). À travers les interactions avec les autres, l'enfant apprend graduellement que son comportement a un impact sur les états mentaux et le comportement d'autrui (Fonagy et collab., 2002). Par contre, des conséquences désastreuses peuvent se produire au niveau du développement affectif et cognitif chez l'enfant lorsque la fonction miroir assurée par la figure d'attachement ne s'exerce pas adéquatement de façon fréquente. Dans cette situation, l'adulte renvoie souvent à l'enfant un message non congruent (p. ex., le reflet que la figure d'attachement donne à l'enfant ne tient pas compte de la détresse vécue 
par ce dernier, la figure d'attachement fait des distorsions importantes lorsqu'elle interprète le comportement de l'enfant, ou encore, elle est plus centrée sur elle-même que sur la détresse vécue par l'enfant) ou non contingent (p. ex., la figure d'attachement néglige l'enfant et ne répond pas à la détresse vécue par ce dernier dans un temps suffisamment rapproché pour que l'enfant puisse associer le comportement de la figure d'attachement à la diminution de sa détresse) avec son expérience interne. Si cela se produit de façon répétée, l'enfant en vient à intégrer un self étranger, composé des représentations mentales de la figure d'attachement qui sont souvent incongrues et déphasées par rapport à la détresse vécue par l'enfant. Le développement d'un self étranger peut engendrer chez l'enfant l'apparition de diverses problématiques telles que les TC (Fonagy et Target, 2006). L'enfant en vient donc à faire des distorsions lorsqu'il interprète les signaux sociaux et les intentions des autres (Dodge, Laird, Lochman et Zelli, 2002; Sharp, 2006; Wied, Goudena et Matthys, 2005). Ces distorsions sont à l'origine des biais de mentalisation positifs ou négatifs qui l'amènent à croire, parfois à tort, que les autres le perçoivent positivement ou négativement (Sharp, 2006; Sharp, Croudace et Goodyer, 2007).

Le développement de la capacité de mentalisation inclut également l'intégration de deux modes de fonctionnement psychique prémentalisants qui sont présents dans la petite enfance: le mode équivalent psychique et le mode comme si (Allen et collab., 2008). Dans le mode équivalent psychique, qui se situe entre 1 an et demi et 3 ans, les états mentaux sont confondus avec la réalité externe; ils ne sont pas perçus comme étant des représentations de celle-ci. L'enfant n'arrive pas encore à comprendre que ce qui se passe dans son esprit est généré par ce dernier et non pas nécessairement par l'environnement réel (Allen et collab., 2008). Par exemple, un enfant croit qu'on ne le voit pas (réalité externe) lorsqu'il ne nous voit pas (réalité interne). Un autre enfant fige et reste muet (réalité interne) lorsqu'il se regarde dans le miroir la première fois alors qu'il porte le costume très réaliste de Batman qu'il a choisi pour l'Halloween (réalité externe) (Domon-Archambault et Terradas, 2012). Dans le mode comme si, vers l'âge de 3 ans, le monde fantasmatique de l'enfant prédomine sur la réalité externe. C'est grâce $\mathrm{au}$ développement du jeu de faire semblant que l'enfant réussit à comprendre qu'il y a une différence entre son monde interne et le monde externe (Allen et collab., 2008). Ces deux mondes se doivent cependant d'être séparés, car la confrontation à la réalité inhibe l'imagination chez l'enfant (Allen et collab., 2008). Lorsque le mode comme si prédomine 
chez l'enfant, il n'est pas encore conscient qu'il fait semblant quand il joue. Par exemple, lorsqu'un enfant joue avec son père à la guerre et que ce dernier prend avec entrain un rouleau de papier essuie-tout pour simuler un fusil, l'enfant peut répondre: «Mais non papa, c'est pas un fusil, c'est un rouleau d'essuie-tout! (Domon-Archambault et Terradas, 2012).

De l'intégration de ces deux modes de fonctionnement psychique résultera la capacité de mentalisation de l'individu (Verheugt-Pleiter, Zevalkink et Schmeets, 2008). C'est vers l'âge scolaire, autour de 5 ou 6 ans, que l'enfant est en mesure de discerner ce que les autres pensent et d'avoir une compréhension nuancée des conduites et des pensées d'autrui (Ensink et Mayes, 2010; Wellman et Lagattuta, 2000). Lorsqu'il est capable d'une telle attribution d'états mentaux, l'enfant est en mesure de donner un sens aux comportements d'autrui, qu'il pourra par la suite anticiper (Fonagy, 1995; Fonagy et Target, 2006). La capacité à comprendre ses propres états mentaux et ceux des autres semblerait être une composante indispensable de l'habileté de l'enfant à identifier les sentiments d'autrui, pour ensuite agir de façon empathique et altruiste selon la situation (Fonagy, 1995; Fonagy et Target, 2006). Ainsi, la mentalisation joue un rôle crucial dans le développement des comportements adaptatifs facilitant le maintien des relations interpersonnelles (Slade, 2006).

\section{L'empathie, la mentalisation et les TC}

L'empathie et la mentalisation permettent à l'enfant de mieux comprendre ses propres comportements et ceux d'autrui. Cela se traduit habituellement par des réponses émotionnelles adaptées à la situation ainsi que par des comportements qui visent à aider les autres. C'est pourquoi l'étude actuelle tentera d'examiner si ces habiletés influencent les comportements externalisés associés aux TC chez l'enfant (p. ex., l'opposition, l'inattention, l'hyperactivité, l'impulsivité, l'agressivité). Cependant, comprendre les variables influençant les TC n'est pas simple. La complexité de la relation entre la capacité à prendre la perspective de l'autre et les comportements agressifs est d'ailleurs mise en lumière dans les travaux de Dodge et ses collègues (2002). Selon ces auteurs, une série de facteurs serait impliquée dans la compréhension des comportements externalisés. La capacité d'être empathique envers autrui serait un des facteurs associés à une faible présence des TC, mais la relation entre l'empathie et ces derniers pourrait varier en fonction 
des situations auxquelles l'enfant est confronté, ce qui laisse supposer la présence d'autres facteurs explicatifs en lien avec les TC (Dodge, 1980; Dodge et Frame, 1982). En ce sens, Goleman (2006) suggère qu'il est plus facile d'être empathique lorsque la situation émotionnelle d'autrui concorde avec celle de l'enfant. Cependant, lorsque la situation d'autrui diffère grandement du vécu de l'enfant, ce dernier a besoin de faire un plus grand effort pour être empathique. C'est dans ces moments que la mentalisation jouerait un rôle important; elle aiderait l'individu à mettre de côté ses propres représentations pour tenter de comprendre celles d'autrui (Allen et collab., 2008). En effet, une personne qui possède une bonne capacité de mentalisation ne présumera pas que les autres pensent ou se sentent comme elle dans une situation donnée. La personne doit donc être en mesure de pouvoir changer sa perspective et d'essayer de prendre le point de vue de l'autre. Une bonne capacité de mentalisation requiert de la flexibilité mentale pour que la personne soit en mesure d'élaborer ce qui pourrait expliquer le comportement d'autrui et ainsi aller au-delà de ce qui est manifeste. Elle implique également l'établissement de liens entre les représentations mentales et la réalité concrète. Ce sont ces derniers liens qui pourraient faire défaut chez les enfants présentant des TC (Allen et collab., 2008) à cause des biais de mentalisation souvent présents chez eux (Sharp, 2006).

De plus, selon Allen et collab. (2008), les enfants présentant des TC peuvent avoir vécu un ou plusieurs traumas, ces expériences de vie pouvant altérer leur capacité de mentalisation. En effet, Allen et ses collègues (2008) suggèrent que les comportements abusifs et violents vécus par les enfants au sein des relations parents-enfant évoquent chez eux de la frustration et de la colère, qui diminueraient à leur tour la capacité de mentalisation. En plus d'occasionner des traumas sévères, la violence augmenterait le risque que la victime s'engage ultérieurement à faire usage de violence envers autrui. Certaines études démontrent que les sentiments présents sont inexorablement influencés par les expériences passées, ce qui influence la capacité de mentalisation. En effet, mentaliser habilement s'avère beaucoup plus difficile lorsque la situation est chargée émotivement, car les capacités de la personne à mentaliser plus objectivement (penser et ressentir clairement) sont affectées (Fonagy et collab., 2002; Jurist, 2005).

Sharp (2006) suggère d'ailleurs que les enfants ayant des TC démontreraient une inhibition de l'empathie plutôt qu'une défaillance réelle au niveau de la capacité à identifier les états mentaux d'autrui. Ils seraient donc en mesure de percevoir et d'organiser leurs propres 
comportements et ceux des autres sous forme d'états mentaux. Cependant, ces enfants auraient une propension à faire des distorsions lorsqu'ils interprètent les signaux sociaux et les intentions des autres (Dodge et collab., 2002; Sharp, 2006; Wied et collab., 2005). Ils auraient davantage tendance à interpréter ces signaux sociaux comme étant plutôt hostiles ou effrayants (Crick et Dodge, 1996; Dodge, 1983; Evans, Heriot et Friedman, 2002), ce qui affecterait leur capacité à manifester une réponse empathique (Lanzetta et Englis, 1989). De plus, ces enfants auraient tendance à présenter souvent des biais de mentalisation positifs les amenant à croire, parfois à tort, que les autres les perçoivent positivement lorsqu'ils manifestent des comportements externalisés inadéquats (Sharp, 2006; Sharp et collab., 2007). Cette tendance est décrite dans les écrits scientifiques plus récents comme étant de la "pseudo-mentalisation " puisque certains éléments essentiels à une identification juste et congruente des états mentaux sont manquants (Allen et collab., 2008). Dans le cas spécifique des TC, la pseudo-mentalisation implique l'usage de la mentalisation à des fins de manipulation ou de contrôle d'autrui, contrairement à la capacité de mentalisation qui reflète une véritable curiosité et un respect pour les états mentaux d'une autre personne (Allen et collab., 2008). La perception erronée d'hostilité chez autrui fait en sorte que l'enfant se sent souvent en danger; l'identification des états mentaux des autres devient ainsi altérée et biaisée chez ces enfants. D'ailleurs, Allen et ses collègues (2008) rapportent qu'une personne doit faire plus d'efforts pour mentaliser lorsqu'elle se sent dans une relation de compétition ou encore ne se sent pas en confiance avec une autre personne.

Pour leur part, Blair, Colledge et Mitchell (2001) considèrent que les enfants qui ont de la difficulté à reconnaître les états mentaux d'autrui à partir de l'expression faciale et du ton de la voix de l'interlocuteur ne sont pas en mesure d'acquérir complètement les habiletés liées à la mentalisation et, par le fait même, d'inhiber leur niveau d'agressivité, pouvant ainsi occasionner certains TC. Tel que démontré par Blair et ses collègues (2001), les enfants présentant des TC manifestent une réponse affective plus faible face aux émotions de peur et de tristesse manifestées par autrui. De plus, ces enfants auraient besoin que les stimuli soient plus intenses pour qu'ils soient en mesure de les reconnaître. Selon Dodge et collab. (2002), les enfants ayant des TC ont tendance à avoir des déficits dans toutes les sphères du processus d'élaboration des informations sociales, soit au niveau de l'encodage de ces informations, de l'interprétation et de la représentation de la 
cause des évènements, de la clarification des résultats attendus lors des interactions sociales et, finalement, dans leurs réponses, décisions et agissements au plan social.

Une différence entre les sexes est également soulevée par Sharp (2006). L'auteure suggère que les filles exprimeraient différemment leur capacité de mentalisation que les garçons. Elles auraient plus tendance à manifester l'agressivité de façon subtile tandis que les garçons recourraient davantage à des comportements externalisés pour exprimer leur agressivité ou leur mal-être. Stetson (1998) suggère un phénomène similaire pour expliquer les différences entre les filles et les garçons quant à la capacité d'empathie. Selon l'auteur, les garçons ne seraient pas nécessairement moins empathiques que les filles, mais ils exprimeraient différemment ou moins ouvertement l'empathie envers autrui, ce qui ferait en sorte qu'ils paraitraient parfois moins empathiques. L'expression par l'agir au détriment de la réflexion, présente chez les garçons, peut être une explication plausible à la surreprésentation de garçons dans les différentes catégories diagnostiques associées aux TC (American Psychiatric Association; APA, 2000) et dans les classes spécialisées en ce type de trouble (Massé, Desbiens et Lanaris , 2006; MÉLS, 2010).

Finalement, plusieurs chercheurs sont d'accord pour dire que la capacité de régulation des émotions d'un individu est essentielle aux manifestations d'empathie (Kozéki et Berghammer, 1992). Par ailleurs, selon Verheugt-Pleiter et collab. (2008), les processus impliqués dans la régulation des émotions sont fortement influencés par la capacité de mentalisation d'un individu. En ce sens, Wied et ses collaborateurs (2005) suggèrent que les enfants impulsifs et agressifs ayant de faibles capacités de régulation des émotions et du comportement, ainsi que de la difficulté à prendre la perspective d'autrui, ne sont pas en mesure de faire passer le bien-être de l'autre avant le leur, ce qui pourrait expliquer en partie leur plus faible taux de gestes pro-sociaux. La mentalisation constituerait un facteur de protection, en ce sens qu'elle permettrait d'inhiber les comportements plus agressifs et ferait en sorte que la personne serait en mesure de freiner ses impulsions et comportements négatifs. Elle agirait comme une sorte de bouton pause (Allen et collab., 2008).

Le niveau d'activation émotionnelle (sous-activation ou suractivation) est également un facteur important qui influence la capacité de mentaliser dans diverses situations (Allen et collab., 2008). D'ailleurs, 
la capacité de mentalisation peut être influencée par l'exposition à un stress. En effet, une personne exposée à un grand niveau d'anxiété peut perdre momentanément sa capacité à réfléchir à propos des pensées et sentiments des autres (Fonagy et collab., 2002). Il est donc possible que le fait de voir une personne en détresse active beaucoup plus facilement les jeunes qui sont moins aptes à gérer émotionnellement tout ce que leur fait vivre cette situation. Cette suractivation émotionnelle pourrait les amener à vouloir soulager leur propre détresse et, par le fait même, à agir de façon apparemment plus égoïste (Kozéki et Berghammer, 1992; Wied et collab., 2005).

Les écrits scientifiques démontrent que les liens entre la mentalisation et la présence des TC chez l'enfant ont maintes fois été étudiés (Allen et collab., 2008; Blair et collab., 2001; Fonagy et collab., 2002; Sharp, 2006; Sharp et collab., 2007). Les liens entre l'empathie et les manifestations des TC ont également fait l'objet de plusieurs recherches (Arsenio et Fleiss, 1996; Hugues, Dunn et White, 1998; Hugues, White, Sharpen et Dunn, 2000). Cependant, les études antérieures ont exploré ces variables séparément. La présente recherche tentera de pallier cette lacune en incluant l'influence que la mentalisation peut avoir sur le développement de l'empathie, des comportements pro-sociaux et des TC.

\section{Objectifs de recherche et hypothèses}

Les TC, l'empathie, les comportements pro-sociaux et les biais de mentalisation ont fait l'objet de plusieurs écrits scientifiques. À notre connaissance, ces variables sont cependant examinées ici conjointement pour la première fois. Cette recherche vise donc à comprendre les liens entre la présence de biais de mentalisation, l'empathie, les comportements pro-sociaux et les manifestations des TC chez les enfants de sexe masculin âgés de 7 à 12 ans. La première hypothèse propose que le niveau d'empathie, la tendance à adopter des comportements pro-sociaux et la présence des biais de mentalisation permettront de prédire le niveau de $\mathrm{TC}$ chez les garçons. La deuxième hypothèse suggère, comme le rapporte Sharp (2006), que les enfants ayant un niveau moins élevé de TC auront une plus grande capacité d'empathie comparativement aux enfants présentant des TC sévères. La troisième hypothèse propose que les enfants ayant des TC pourraient utiliser leur capacité d'empathie de façon négative à cause de leur interprétation parfois erronée des situations sociales (Sharp, 2006; Crick et Dodge, 
1996; Dodge, 1983; Evans et collab., 2002; Lanzetta et Englis, 1989), ce qui serait lié à la présence des biais de mentalisation et à la diminution des comportements pro-sociaux.

\section{Méthode}

\section{Participants}

Cinquante et un garçons âgés de 7 à 12 ans, présentant des TC à divers degrés, issus de différentes écoles de la région de Montréal et ses environs, et leurs parents, ont participé à l'étude. La présence et le degré de TC chez les enfants ont été vérifiés à l'aide du Questionnaire de forces et de difficultés de l'enfant (Strenght and Difficulties Questionnaire; Goodman, 1997) complété par le parent. Les enfants présentant une déficience intellectuelle ou un trouble du spectre de l'autisme, préalablement identifiés par les professionnels des écoles participant à l'étude, ont été exclus de l'échantillon, ces enfants pouvant présenter des défaillances importantes au niveau des capacités d'empathie et de mentalisation (Midgley et Vrouva, 2012).

\section{Procédure}

Premièrement, l'approbation du comité d'éthique de la recherche Lettres et sciences humaines de l'Université de Sherbrooke a été obtenue. Deuxièmement, les directions des écoles concernées par l'étude ont été rencontrées afin de leur expliquer la nature, les avantages et les inconvénients de la participation des enfants et des parents à la recherche. Troisièmement, un dépliant décrivant l'étude a été envoyé à tous les parents des garçons âgés de 7 à 12 ans des écoles ayant accepté de participer à la recherche. Quatrièmement, le formulaire d'information et de consentement ainsi que les questionnaires devant être remplis par les parents ont été envoyés à ceux ayant accepté de participer à l'étude. Enfin, après réception des formulaires et questionnaires remplis par les parents, les questionnaires destinés aux enfants ont été administrés en groupe dans leurs écoles respectives. 


\section{Instruments de mesure ${ }^{1}$}

\section{Mesures de la capacité d'empathie de l'enfant.}

Mesure d'empathie de Griffith (GEM; Dadds et collab., 2008). Ce questionnaire, rempli par un des parents, est une adaptation du Bryant's Empathy Index (Bryant, 1982) et a été validé auprès de parents d'enfants âgés de 4 à 16 ans (Dadds et collab., 2008). L'instrument est composé de 23 items. Le parent doit répondre à l'aide d'une échelle de Likert variant de -4 à 4 . Les résultats du questionnaire donnent trois scores, soit un score global, un score d'empathie cognitive et un score d'empathie affective. Linstrument démontre une bonne convergence avec des questionnaires autorapportés par les enfants mesurant l'empathie. Le GEM démontre également une bonne fidélité test-retest et une bonne cohérence interne pour les différents groupes d'âge et les deux sexes (Dadds et collab., 2008).

Échelle d'empathie élémentaire (D'Ambrosio, Olivier, Davina et Besche, 2009). Ce questionnaire constitue une traduction en français de la Basic Empathy Scale (BES), développée par Jolliffe et Farrington (2006). Linstrument est composé de 20 items à échelle de type Likert variant de 0 à 4 . Rempli par les enfants, ce questionnaire mesure, à l'aide de deux sous-échelles, les composantes cognitives et affectives de l'empathie. La version française du BES possède des qualités psychométriques satisfaisantes (D'Ambrosio et collab., 2009). La fidélité test-retest de l'échelle concernant la composante cognitive est de 0,66 tandis que celle de l'échelle qui a trait à la composante affective est de 0,77 (D'Ambrosio et collab., 2009).

Dans le contexte de la présente étude, un score combiné de la capacité d'empathie a été utilisé pour faire les analyses statistiques. Ce score

\footnotetext{
${ }^{1 .}$ La mesure d'empathie de Griffith (Griffith Empathy Measure; Dadds et collab., 2008), la Tâche de mentalisation (Mentalizing Task; Sharp et collab., 2007) et les Tendances pro-sociales (Prosocial Tendencies Measure; Carlo et Randall, 2002) ont été traduites selon la méthode de traduction/retraduction (Behling et Law, 2000) en utilisant une approche consensuelle (Bradley, 1994) par Girard, Terradas et Achim (2012b, 2012c). Selon cette méthode, deux personnes bilingues produisent chacune une traduction de façon indépendante, puis comparent leurs versions et résolvent les différences dans le texte. Une troisième personne retraduit ensuite l'instrument dans sa langue d'origine afin de s'assurer que les énoncés ont conservé leur sens. La traduction est finalement révisée par un comité d'experts et d'utilisateurs potentiels de l'entrevue. Pour sa part, la version en français de l'Échelle d'empathie élémentaire (D'Ambrosio et collab., 2009; Basic Empathy Scale; Jolliffe et Farrington, 2006) a été adaptée au contexte québécois par Girard, Terradas et Achim (2012a).
} 
combiné, qui tient compte autant de la perception de l'enfant que de celle du parent, a été calculé en faisant la moyenne des scores globaux standardisés des deux instruments qui mesurent la capacité d'empathie de l'enfant afin d'obtenir un seul score global du niveau d'empathie de chaque participant. De par sa force $(0,87)$, l'alpha de Cronbach global démontre d'ailleurs la pertinence de ce choix.

\section{Mesure des biais de mentalisation de l'enfant}

Tâche de mentalisation (Sharp et collab., 2007). Cet instrument permet d'évaluer les biais de mentalisation chez les enfants d'âge scolaire. Elle consiste en une série de 15 vignettes, écrites et illustrées sur des planches, portant sur diverses situations sociales qui font appel à la capacité de mentalisation de l'enfant. Ces situations concernent la solitude, la peur d'être ridiculisé, la gêne en contexte social, le rejet, le fait d'être peu performant en sport, le fait d'avoir un accident, la perte, le fait d'être seul, l'échec scolaire, le déménagement, la taille physique, les handicaps, l'embarras social, le divorce et la pauvreté. Pour chaque vignette, l'enfant a trois choix de réponse qui correspondent à trois styles différents d'attribution d'états mentaux, soit un biais positif irréaliste avec une forte référence à l'image de soi, soit un biais négatif irréaliste avec une forte tendance à l'autodépréciation, soit une réponse neutre démontrant un style d'attribution plutôt rationnel et adaptatif. Le style d'attribution prédominant chez l'enfant est déterminé en fonction du plus grand nombre de réponses qu'il obtient à l'un ou l'autre des trois styles. Le temps nécessaire pour y répondre est d'environ 20 minutes. L'instrument comporte une bonne validité de construit; les biais de mentalisation sont positivement corrélés aux difficultés de comportements externalisés chez les enfants d'âge scolaire (Sharp et collab., 2007). Dans le contexte de la présente étude, un score combiné de biais de mentalisation, représentant le nombre total de biais positifs et négatifs, a été créé. L'indice de cohérence interne obtenu dans cette recherche est satisfaisant $(0,71)$.

\section{Mesures des comportements de l'enfant}

Troubles de comportement. Afin de mesurer la présence des TC chez l'enfant, la version française du Strengths and Difficulties Questionnaire (Goodman, 1997) a été administrée à un des parents. L'instrument est composé de 25 items selon une échelle de Likert variant de 0 à 2 . Seule l'échelle relative aux TC a été utilisée afin de vérifier la présence chez les enfants des indicateurs cliniques liés à ces troubles. Cette échelle mesure 
certaines manifestations des TC telles que le niveau de mensonges, de vols, de comportements agressifs (bagarres), de désobéissance et de difficultés de régulation émotionnelle (crises de colère). Le temps nécessaire pour répondre au questionnaire est d'environ 20 minutes. Lindice de validité convergente avec les symptômes des TC répertoriés dans la quatrième édition révisée du Manuel diagnostique et statistique des troubles mentaux (DSM-IV-TR; APA, 2000) est satisfaisant (Sharp et collab., 2007). La validité convergente avec la Liste de vérification du comportement des jeunes de 6 à 18 ans (CBCL; Achenbach et Rescorla, 2001), un instrument fréquemment utilisé en clinique et en recherche afin de vérifier la présence chez les enfants de troubles externalisés, est également satisfaisante (Goodman, 1997). Les indices de fidélité test-retest de l'instrument oscillent entre 0,80 et 0,82 . La cohérence interne du questionnaire est aussi satisfaisante (Sharp et collab., 2007). Dans cette étude, l'indice de cohérence interne de l'échelle des TC est également satisfaisant $(0,70)$.

Tendances pro-sociales. Remplie par un des parents, la traduction française du questionnaire Prosocial Tendencies Measure (Carlo et Randall, 2002) est composée de 24 items à échelle de Likert variant de 0 à 4 . Six types de comportements pro-sociaux sont mesurés dans ce questionnaire: publics, anonymes, déployés dans des situations de sinistre, émotifs, manifestés par complaisance et de nature altruiste. Les résultats sont compilés pour donner un score global de la tendance de l'enfant à manifester des comportements pro-sociaux variant de 0 à 96. En l'absence d'un seuil clinique et de normes de comparaison, le score total de l'enfant représente la somme des comportements prosociaux manifestés par celui-ci. Plus un enfant présente un score élevé, plus il aura tendance à manifester des comportements pro-sociaux. Les recherches de Carlo et Randall (2002) démontrent des indices de cohérence interne variant entre 0,59 et 0,86 , avec une fidélité test-retest de l'échelle globale de 0,67 . Lindice de cohérence interne de l'étude actuelle est excellent $(0,95)$.

\section{Résultats}

D'abord, des analyses préliminaires des données ont permis de vérifier que les variables à l'étude respectent les postulats de base (normalité, asymétrie, aplatissement et données extrêmes) (voir le tableau 1). Ensuite, des corrélations de Pearson ont été effectuées afin de mieux définir les relations entre les TC, l'empathie, les biais de mentalisation 
et les comportements pro-sociaux (voir le tableau 2). Les résultats de ces analyses suggèrent que l'âge de l'enfant est corrélé négativement avec le score combiné des biais de mentalisation (score total des biais de mentalisation positifs et négatifs) $(r=-0,45, p=0,01)$ et les biais de mentalisation positifs $(r=-0,42, p=0,05)$. Ainsi, plus les enfants vieillissent, moins ils ont tendance à avoir ces types de biais. Cependant, la présence de biais de mentalisation négatifs ne semble pas changer en fonction de l'âge de l'enfant, ce qui suggère que seuls les biais de mentalisation positifs diminuent au fur et à mesure que les enfants vieillissent.

Tableau 1 - Moyenne, écart-type et étendue pour toutes les variables

\begin{tabular}{lccc}
\hline Variables & Moyenne & Écart-type & Étendue \\
\hline Âge & 9,55 & 1,40 & $7-12$ \\
Comportements pro-sociaux & 72,29 & 17,14 & $23-96$ \\
Empathie cognitive (version enfant) & 23,25 & 5,62 & $10-32$ \\
Empathie affective (version enfant) & 21,51 & 6,41 & $8-35$ \\
Empathie totale (version enfant) & 44,76 & 8,86 & $23-64$ \\
Empathie cognitive (version parent) & 359,61 & 263,51 & $5-783$ \\
Empathie affective (version parent) & 371,45 & 220,44 & $1-667$ \\
Empathie totale (version parent) & 431,43 & 200,35 & $4-665$ \\
Niveau de TC & 3,10 & 2,31 & $0-8$ \\
Présence TC ou pas & 0,45 & 0,50 & $0-1$ \\
Empathie combinée & 0,00 & 1,39 & $-3,05-2,53$ \\
Biais de mentalisation combiné & 0,28 & 0,45 & $0-1$ \\
Biais de mentalisation positif & 3,33 & 2,63 & $0-11$ \\
Biais de mentalisation négatif & 3,49 & 3,56 & $0-14$ \\
\hline
\end{tabular}




\section{Tableau 2 - Corrélations entre les différentes variables de l'étude}

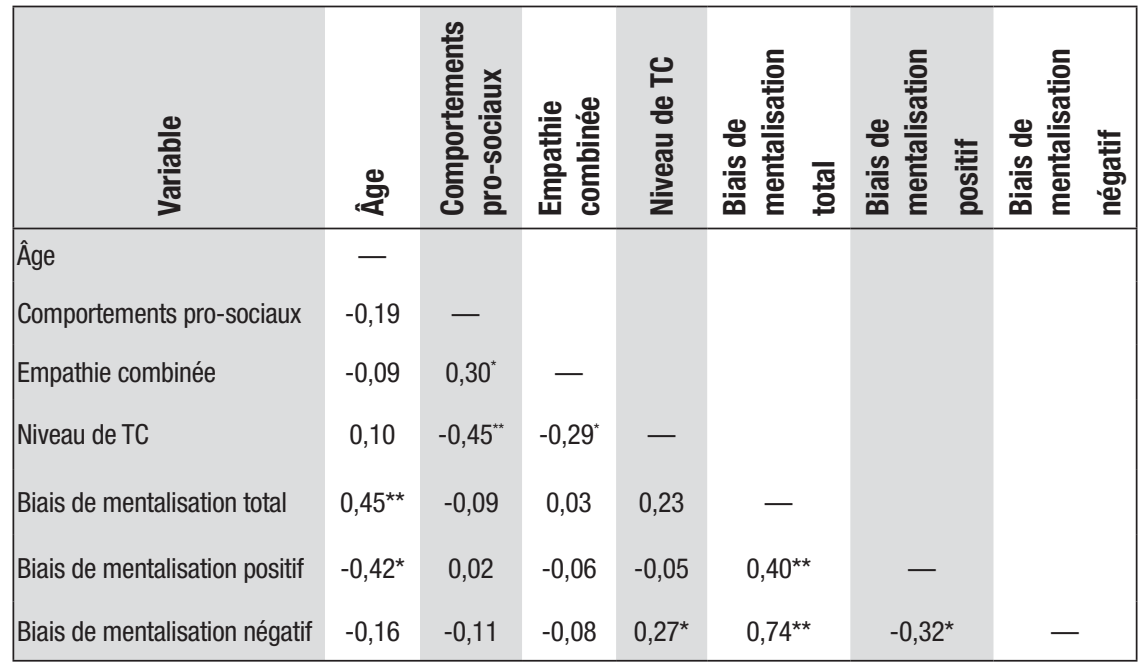

$* p<0,05 ; * * p<0,01$

La première hypothèse proposait que le niveau d'empathie, la tendance à adopter des comportements pro-sociaux et la présence des biais de mentalisation pourraient permettre de prédire le niveau de TC chez les garçons. Des analyses de régressions multiples ont été utilisées afin d'examiner cette hypothèse (voir le tableau 3). L'objectif de ces analyses était de vérifier la contribution spécifique de l'empathie, des comportements pro-sociaux et des biais de mentalisation à la prédiction des TC au-delà de l'effet des autres variables ainsi que de connaître le pourcentage de la variance des TC que l'empathie, les biais de mentalisation et les comportements pro-sociaux expliquent une fois combinés. Les résultats des analyses de régressions multiples démontrent que l'empathie, les biais de mentalisation négatifs et les comportements pro-sociaux expliquent $27,7 \%$ de la variance totale des TC. En analysant les résultats de plus près, il est possible d'observer que l'empathie n'est plus associée de façon significative aux TC, indiquant ainsi qu'il pourrait y avoir un processus de médiation à l'œuvre ou que, tout simplement, l'empathie n'est plus une variable aussi déterminante comparativement aux autres, car son effet disparait quand celui des autres est pris en considération. Les biais de mentalisation négatifs ainsi que les comportements pro-sociaux contribuent donc de façon unique aux manifestations de TC chez l'enfant. En effet, lorsque les 
analyses de régression sont faites sans la variable de l'empathie, les biais de mentalisation négatifs ainsi que les comportements pro-sociaux expliquent encore $24,5 \%$ de la variance totale des TC.

\section{Tableau 3 - Analyses de régression incluant les biais de mentalisation négatifs, l'empathie et les comportements pro-sociaux comme prédicteurs des TC}

\begin{tabular}{llcc}
\hline Variable prédite & Prédicteurs & $\boldsymbol{\beta}$ & $\mathbf{R}^{2}$ \\
\hline $\begin{array}{l}\text { Troubles de } \\
\text { comportement }\end{array}$ & Empathie & $-0,16$ & $27,7 \%$ \\
& $\begin{array}{l}\text { Biais de mentalisation } \\
\text { négatif }\end{array}$ & $-0,38^{*}$ & \\
& $\begin{array}{l}\text { Comportements } \\
\text { pro-sociaux }\end{array}$ & $0,22^{\star}$ & \\
& & & \\
\hline
\end{tabular}

$* p<0,05$

La deuxième hypothèse suggérait que, comme le rapporte Sharp (2006), les jeunes ayant un niveau moins élevé de TC auraient une plus grande capacité d'empathie comparativement aux enfants présentant des TC sévères. Les analyses de corrélation démontrent que les TC et les comportements pro-sociaux sont associés entre eux de façon négative $(r=-0,45, p=0,01)$. Ainsi, plus un enfant a des TC, moins il aurait tendance à manifester des comportements pro-sociaux envers autrui. De plus, les analyses de corrélation soulèvent que la capacité d'empathie de l'enfant est associée négativement à la présence des TC $(r=-0,29$, $p=0,05)$ et positivement aux manifestations des comportements prosociaux $(r=0,30, p=0,05)$. Ces deux dernières variables sont également associées négativement entre elles $(r=-0,45, p=0,01)$. Ces résultats démontrent que plus les garçons d'âge scolaire ont une bonne capacité d'empathie, moins ils ont un niveau élevé de TC et plus ils manifestent des comportements pro-sociaux.

Finalement, la troisième hypothèse retenue proposait que les enfants ayant des TC pourraient se servir de cette habileté de façon négative à cause de leur interprétation parfois erronée des situations sociales, ce qui serait lié à la présence des biais de mentalisation et à la diminution des comportements pro-sociaux. En ce sens, les analyses démontrent que les biais de mentalisation positifs et totaux ne sont pas corrélés significativement avec le niveau de TC. Cependant, les 
TC sont corrélés positivement de façon significative avec les biais de mentalisation négatifs $(r=0,27, p=0,05)$. Ceci suggère que, lorsque les jeunes garçons ont tendance à avoir des biais de mentalisation négatifs, leur niveau de TC est plus élevé. Ces résultats répondent partiellement à l'hypothèse 3 , car un lien avec les comportements pro-sociaux n'est pas directement observé.

\section{Discussion}

L'objectif principal de cette étude était de mieux comprendre les liens entre la capacité d'empathie, les biais de mentalisation, les comportements pro-sociaux et les TC chez les garçons âgés de 7 à 12 ans. Les résultats de l'étude ont tout d'abord permis de découvrir que l'âge de l'enfant corrèle négativement avec la présence des biais de mentalisation positifs qui sont reliés à une forte propension chez l'enfant à penser que les autres le perçoivent de façon positive. Donc, plus les enfants avancent en âge, moins ils ont tendance à présenter des biais de mentalisation positifs. Cependant, les biais de mentalisation négatifs, associés à une forte propension chez l'enfant à penser que les autres le perçoivent de façon négative, ne changent pas en fonction de l'âge de l'enfant. Comme il a été suggéré plus haut dans les hypothèses, il était attendu que les enfants ayant des TC pourraient se servir de leur capacité de mentalisation de façon négative à cause de leur interprétation parfois erronée des situations sociales, ce qui serait lié à la présence des biais de mentalisation. La stabilité et la cristallisation dans le temps de ces biais pourraient avoir des effets néfastes pour les enfants présentant des TC. Ainsi, les enfants ayant une propension à penser que les autres sont hostiles à leur égard peuvent accumuler de la colère et du ressentiment envers autrui, sentiments qui pourraient s'exprimer par de l'agressivité ou des comportements inadéquats. Les jeunes auraient donc fréquemment l'impression qu'ils sont menacés par leur environnement et qu'ils doivent nécessairement se défendre, renforçant ainsi leur nécessité à avoir recours à des biais de mentalisation. Une autre particularité de l'étude est que le nombre total de biais de mentalisation positifs semble augmenter avec l'âge lorsque l'enfant présente un certain niveau de TC. Ceci peut s'expliquer par une certaine cristallisation du mode prédominant de mentalisation chez l'enfant à travers le temps, suggérant ainsi la nécessité de prévenir une augmentation des biais de mentalisation en intervenant tôt auprès de ces jeunes. 
Les résultats révèlent également que la capacité d'empathie des jeunes garçons est fortement corrélée avec la tendance observée chez ceux-ci à manifester des comportements pro-sociaux. De plus, selon les résultats de cette étude, les TC et les comportements pro-sociaux seraient associés entre eux de façon négative. En effet, ces résultats appuient ceux de Sharp (2006), qui stipulent que les jeunes ayant un niveau moins élevé de TC auraient de plus grandes capacités empathiques comparativement aux enfants présentant des TC sévères. Les résultats de la présente étude semblent également en lien avec ceux des recherches précédentes qui ont en effet constaté que l'empathie serait un acquis développemental essentiel à l'accomplissement des gestes pro-sociaux envers autrui (Eisenberg et collab., 2006; Hoffman, 2001; Krebs et Van Hesteren, 1994). Les résultats de l'étude démontrent également qu'un jeune qui est prédisposé à manifester des comportements pro-sociaux aura moins tendance à présenter des comportements externalisés dans son quotidien (Greenberg, Kusche et Speltz, 1991; Vitaro et Gagnon, 2008). En effet, si on interprète ces résultats, il est possible de voir que les comportements pro-sociaux pourraient être une variable médiatrice entre l'empathie et les $\mathrm{TC}$, comme le suggèrent les résultats de Girard, Terradas et Matté-Gagné (soumis). Ces résultats peuvent ainsi être le point de départ d'une réflexion concernant les difficultés sociales éprouvées par les enfants présentant des TC. Selon Stetson (1998), ressentir des émotions similaires à celles d'autrui peut être la motivation principale pour qu'un enfant réponde d'une manière prosociale dans une situation donnée. Par contre, si l'enfant n'est pas en mesure de comprendre le point de vue d'autrui, il peut s'avérer difficile pour lui de trouver une action qui pourrait être aidante pour l'autre. Ceci veut donc dire que l'enfant a besoin de bien développer les processus cognitifs et affectifs qui sont tous deux nécessaires à une compréhension plus complète de l'expérience d'autrui. Ce développement pourrait ainsi diminuer la probabilité que l'enfant manifeste un comportement inadéquat (Stetson, 1998).

Il était également attendu que les TC puissent être prédits par le niveau d'empathie, les comportements pro-sociaux et les biais de mentalisation négatifs. L'hypothèse ainsi confirmée laisse croire que certaines de ces variables, soit les comportements pro-sociaux et les biais de mentalisation négatifs, prédisent $27,7 \%$ des TC chez les jeunes garçons lorsqu'elles sont concomitantes. Ceci porte donc à réfléchir sur le rôle que joue l'empathie en lien avec les TC. Une partie de l'équation pourrait être expliquée par le lien de médiation trouvé par Girard et 
collab., (soumis) lors d'une recherche précédente. Les résultats de cette étude suggèrent un effet indirect de l'empathie sur les TC qui dépend de la présence chez l'enfant des comportements pro-sociaux. L'empathie n'aurait donc pas une influence directe sur les TC, mais elle aurait pour rôle d'entrainer en premier lieu les garçons à aider leurs proches, ce qui aurait pour conséquence d'influencer l'intensité des TC chez ceuxci. L'empathie aurait donc un lien important avec la tendance à agir des comportements pro-sociaux. Ainsi, il est possible de penser que des lacunes sur le plan des comportements pro-sociaux pourraient être liées aux difficultés de ces enfants à bien interpréter les signaux sociaux (Bretherton, Fritz, Zahn-Waxler et Ridgeway, 1986; Dodge et collab., 2002; Mize et Pettit, 2008), engendrant ainsi des confusions et des conflits lors des interactions avec les pairs et les adultes qui les entourent.

L'étude actuelle soulève également l'absence de lien entre l'empathie et les biais de mentalisation. Ceci peut être expliqué par le fait que l'empathie peut être corrélée aux capacités de mentalisation d'une personne, mais pas nécessairement aux biais de mentalisation que celle-ci démontre. La capacité de mentalisation pourrait en effet être nécessaire à l'apparition de l'empathie; cependant, il est possible que les biais de mentalisation viennent influencer cette relation. En effet, les enfants présentant des TC ont plus de difficultés à réguler leurs émotions, notamment lorsqu'ils sont impliqués émotivement dans une situation. Il est donc possible que cela altère leur potentiel réel en lien avec les capacités de mentalisation et d'empathie. Le type de situation, les expériences de vie antérieures et le niveau d'implication émotionnelle dans une situation pourraient moduler la relation entre l'empathie et la mentalisation, soulignant l'importance d'approfondir les recherches à ce sujet.

Il serait aussi important de bien distinguer les types de biais de mentalisation et leur lien avec l'empathie et les TC, car cette étude démontre que ce sont les biais de mentalisation négatifs qui sont associés aux manifestations des TC. Ces résultats ne correspondent pas à ceux de l'étude de Sharp (2006) qui conclut que les enfants présentant des TC manifesteraient des biais de mentalisation positifs dans certaines situations. Ceci les amènerait à mal interpréter certains évènements, altérant ainsi leur capacité à manifester des comportements prosociaux dans des situations données. C'est pourquoi il serait pertinent de reproduire cette étude auprès d'un plus grand échantillon afin de valider les résultats obtenus. 
Bref, les résultats de la présente recherche laissent sous-entendre que les comportements pro-sociaux exprimés dans le but d'aider les autres, mis en lien avec l'absence de biais de mentalisation négatifs, pourraient influencer les manifestations des TC chez les enfants.

L'empathie, quant à elle, engendrerait une tendance chez l'enfant à manifester plus de comportements pro-sociaux, ce qui aurait également pour effet de diminuer les comportements problématiques (Girard et collab., soumis). Donc, l'absence de biais de mentalisation et les comportements pro-sociaux des jeunes garçons jouent un rôle direct dans le maintien des relations sociales plus harmonieuses. Pour sa part, l'empathie jouerait un rôle indirect par rapport aux TC; elle favoriserait les manifestations des comportements pro-sociaux, lesquels contribueraient à diminuer la présence des TC chez l'enfant. Ainsi, le fait qu'une personne présente peu de biais de mentalisation lui permettrait d'entretenir des relations enrichissantes et profondes et d'avoir une meilleure compréhension d'elle-même et, par conséquent, une meilleure estime de soi (Allen et collab., 2008). Il serait alors important de miser sur le développement de ces habiletés chez les jeunes afin d'augmenter leur niveau de relations sociales positives et saines.

\section{Limites}

Les limites de la présente étude doivent être considérées pour les recherches futures. Premièrement, son échantillon restreint limite les possibilités de généralisation des résultats. Pour les recherches futures, il pourrait être intéressant d'avoir un échantillon plus représentatif de la population générale de la province de Québec en termes d'âge et de niveaux des TC des participants. Deuxièmement, les filles ont été exclues de l'étude sur la base des résultats des recherches précédentes qui stipulent que les garçons sont surreprésentés dans la population d'enfants présentant des TC. Pourtant, il serait intéressant et instructif de reproduire l'étude auprès de jeunes filles, ce qui permettrait de vérifier si les mêmes relations entre les comportements pro-sociaux, les biais de mentalisation et l'empathie sont présentes chez elles ou si ces relations diffèrent. Troisièmement, dans la présente étude, seuls les enfants et les parents ont été conviés à participer à la recherche. Pour les études futures, il pourrait être intéressant de solliciter également la participation des enseignants, puisque la capacité d'empathie et les manifestations des comportements pro-sociaux des enfants peuvent s'exprimer différemment à l'école et à la maison (Kozéki et 
Berghammer, 1992; Strayer et Roberts, 1989). Enfin, la façon dont la mentalisation a été mesurée dans cette étude a pu avoir un impact sur la force et la direction des liens observés entre les variables. Lors de recherches futures, il pourrait être intéressant de mesurer la capacité de mentalisation des enfants au lieu d'évaluer les biais de mentalisation de ceux-ci.

\section{Conclusion}

Les résultats de cette recherche démontrent l'importance d'étudier les facteurs potentiellement impliqués dans le développement des TC lorsqu'on considère leur impact sur le fonctionnement de l'enfant. Pour la première fois, selon la connaissance des auteurs, l'empathie et les biais de mentalisation ont été examinés conjointement. Les résultats ont révélé que les liens unissant ces variables permettent une meilleure compréhension des difficultés des jeunes garçons présentant des TC. Deux facteurs de risque reliés aux manifestations des TC ont été identifiés, soit les biais de mentalisation négatifs et les difficultés à adopter des comportements pro-sociaux. Il est donc primordial d'intervenir pour aider les enfants à corriger leurs biais de mentalisation et adopter des comportements pro-sociaux. Certains programmes existants se sont d'ailleurs avérés efficaces pour réduire les TC. Ajouter un volet incluant les biais de mentalisation et un entraînement aux habiletés sociales pourrait s'avérer une avenue intéressante qui mériterait d'être investiguée davantage.

\section{Références}

Achenbach, T. M. et Rescorla, L. A. (2001). Manual for the ASEBA school-age forms et profiles. Burlington, Vermont: University of Vermont, Research Center for Children, Youth et Families.

Alessandri, G., Caprara, G. V., Eisenberg, N. et Steca, P. (2009). Reciprocal relations among self-efficacy beliefs and prosociality across time. Journal of Personality, 77(4), 1229-1259.

Allen, J. G., Fonagy, P. et Bateman, A. W. (2008). Mentalizing in clinical practice. Arlington, VA: American Psychiatric Publishing, Inc.

American Psychiatric Association. (2000). DSM-IV-TR:Manuel diagnostique et statistique des troubles mentaux, texte révisé (4 éd.) (Washington, D.C, 2000). Traduction française par J. D. Guelfi et collab., Paris, France: Masson.

Arsenio, W. et Fleiss, K. (1996). Typical and behaviourally disruptive children's understanding of the emotional consequences of socio-moral events. British Journal of Developmental Psychology, 14, 173-186. 
Barkley, R. A. (1997). Behavioral inhibition, sustained attention, and executive functions: Constructing a unifying theory of ADHD. Psychological Bulletin, 121(1), 65-94. doi: 10.1037/0033-2909.121.1.65

Barkley, R. A. (2006). Attention-deficit hyperactivity disorder. A handbook for diagnosis and treatment ( $3^{\mathrm{e}}$ éd.). New York: Guilford Press.

Baron-Cohen, S. et Wheelwright, S. (2004). The empathy quotient: An investigation of adults with Asperger syndrome or high functioning autism, and normal sex differences. Journal of Autism and Developmental Disorders, 34(2), 163-175.

Batson, C. D. (1998). Altruism and prosocial behaviour. Dans D. T. Gilbert, S. T. Fiske et G. Lindzey (Éds.), Handbook of social psychology (vol. 2, p. 282-316). Boston: McGraw-Hill.

Baving, L., Rellum, T., Laucht, M. et Schmidt, M. H. (2006). Children with oppositional-defiant disorder display deviant attentional processing independent of ADHD symptoms. Journal of Neural Transmission 113(5), 685-693. doi: 10.1007/s00702-005-0345-x

Behling, O. et Law, K. S. (2000). Translating questionnaires and other research instruments: Problems and solutions. London: Sage Publications.

Bjorkqvist, K. et Osterman, K. (2000). Social intelligence, empathy, aggression? Aggression and Violent Behavior, 5, 191-200.

Blair, R. J. R., Colledge, E. et Mitchell, D. G. V. (2001). Somatic markers and response reversal: Is there orbitofrontal cortex dysfunction in boys with psychopathic tendencies? Journal of Abnomal Child Psychology, 29, 499511.

Blair, R. J. R., Peschardt, K. S., Budhani, S., Mitchell, D. G. V. et Pine, D. S. (2006). The development of psychopathy. Journal of Child Psychology and Psychiatry, 7(3-4), 262-275.

Bradley, C. (1994). Handbook of psychology and diabetes: A guide to psychological measurement in diabetes research and practice. Switzerland : Harwood Academic Publisher.

Bretherton, I., Fritz, J., Zahn-Waxler, C. et Ridgeway, D. (1986). Learning to talk about emotions: A functionalist perspective. Child Development, $56,529-548$.

Bryant, B. K. (1982). An index of empathy for children and adolescents. Child Development, 53, 413-425.

Carlo, G. et Randall, B. (2002). The development of a measure of prosocial behaviors for late adolescents. Journal of Youth and Adolescence, 31(1), 31-44.

Chevalier, N. (2010). Les fonctions exécutives chez l'enfant: concepts et développement. Canadian Psychology, 51(3), 149-163.

Cialdini, R. B., Brown, S. L., Lewis, B. P., Luce, C. N. et Steven, L. (1997). Reinterpreting the empathy-altruism relationship: When one into one equals oneness. Journal of Personality and Social Psychology, 73 (3), 481-494. 
Crick, N. R. et Dodge, K.A. (1996). Social information-processing mechanisms on reactive and proactive aggression. Child Development, 67, 993-1002.

D'Ambrosio, F., Olivier, M., Davina, D. et Besche, C. (2009). The basic empathy scale: A french validation of a measure of empathy in youth. Personality and Individual Differences, 46(2), 160-165.

Dadds, M. R., Hunter, K., Hawes, D. J., Frost, A. D. J., Vassallo, S., Bunn, P., Merz, S. et El Masry, Y. (2008). A measure of cognitive and affective empathy in children using parents ratings. Child Psychiatry and Human Development, 39, 111-122.

De Waal, F. B. M. (2008). Putting the altruism back into altruism: The evolution of empathy. Annual Review of Psychology, 59, 279-300.

Dodge, K. A. (1980). Social cognition and children's aggressive behavior. Child Development, 51, 162-170.

Dodge, K. A. (1983). Social-cognitive mechanisms in the development of conduct-disorder and depression. Annual Review of Psychology, 44, 559584.

Dodge, K. A. et Frame, C. L. (1982). Aggressive boys. Child Development, 53, 620-635.

Dodge, K. A., Laird, R., Lochman, J. E. et Zelli, A. (2002). Multidimensional latent-construct analysis of children's social information processing patterns: Correlations with aggressive behavior problems. Psychological Assessment, 14(1), 60-73.

Domon-Archambault, V. et Terradas, M. M. (2012). Manuel de formation aux interventions axées sur la mentalisation destiné aux éducateurs des centres jeunesse. Document inédit. Université de Sherbrooke, Canada.

Eisenberg, N., Fabes, R. A. et Spinrad, T. L. (2006). Prosocial behaviour. Dans W. Damon, R. M. Lerner et N. Eisenberg (dir.), Handbook of child psychology: Social, emotional, and personality development (6 éd., vol. 3, pp. 646-718). New York: Wiley.

Ensink, K. et Mayes, L. C. (2010). The development of mentalisation in children from a theory of mind perspective. Psychoanalytic Inquiry, 30, 301-337.

Evans, I. M., Heriot, S. A. et Friedman, A. G. (2002). A behavioural pattern of irritability, hostility, and inhibited empathy in children. Clinical Child Psychology and Psychiatry, 7, 211-224.

Fabes, R. A., Eisenberg, N. et Eisenbud, L. (1993). Behavioral and physiological correlates of children's reactions to others in distress. Developmental Psychology, 29(4), 655-663.

Fabes, R. A., Eisenberg, N., Karbon, M., Troyer, D. et Switzer, G. (1994). The relations of children's emotion regulation to their vicarious emotional responses and comforting behaviors. Child Development, 65(6), 16781693. 
Fonagy, P. (1995). Playing with reality: The development of psychic reality and its malfunction in borderline personalities. International Journal of Psychoanalysis, 76, 39-45

Fonagy, P. (2003). Toward a developmental understanding of violence. British Journal of Psychiatry, 183, 190-192.

Fonagy, P., Gergely, G., Jurist, E. L. et Target, M. (2002). Affect regulation, mentalization, and the development of the self. New York: Other Press.

Fonagy, P., Steele, M., Steele, H., Moran, G. S. et Higgitt, A. C. (1991). The capacity for understanding mental states: The reflective self in parent and child and its significance for security of attachment. Infant Mental Health Journal, 12(3), 201-219.

Fonagy, P. et Target, M. (2006). The mentalization-focused approach to self pathology. Journal of Personality Disorders, 20(6), 544-576.

Fortin, L., Marcotte, D., Royer, E. et Potvin, P. (2000). Les facteurs discriminants sur les plans personnel, familial et scolaire entre les troubles du comportement intériorisés, extériorisés et concomitants chez des élèves de première secondaire. Revue des sciences de l'éducation, 26, 197-218.

Girard, E., Terradas, M. M. et Achim, J. (2012a). Griffith Empathy Measure: traduction française. D'après la version originale de M. R. Dadds, K. Hunter, D. J. Hawes, A. D. J. Frost, S. Avassallo, P. Bunn, S. Merz et Y. El Masry (2007). Document inédit. Université de Sherbrooke, Canada.

Girard, E., Terradas, M. M. et Achim, J. (2012b). Prosocial Tendencies Measure: traduction française. D’après la version originale de G. Carlo et B. Randall (2002). Document inédit. Université de Sherbrooke, Canada.

Girard, E., Terradas, M. M. et Achim, J.(2012c).Mentalizing Task:traduction française. D'après la version originale de C. Sharp, T. J. Croudace et I. M. Goodyer (2007). Document inédit. Université de Sherbrooke, Canada.

Girard, E., Terradas, M. M. et Matte-Gagné, C. (soumis). Empathie, comportements pro-sociaux et troubles de comportement chez les enfants. Enfance.

Goleman, D. (2006). Social intelligence: The new science of human relationships. New York, NY: Bantam Books.

Goodman, R. (1997). The Strengths and Difficulties Questionnaire: A research note. Journal of Child Psychology and Psychiatry, 38, 581-586.

Greenberg, M. T., Kusche, C. A., Speltz, M. (1991). Emotional regulation, self-control, and psychopathology : The role of relationships in early childhood. Dans D. Cicchetti et S. L. Toth (dir.), Internalizing and externalizing expressions of dysfunction (pp. 21-55). Hillsdale, New Jersey : Lawrence Erlbaum Associates, Inc.

Greene, R. W. et Doyle, A. E. (1999). Toward a transactional conceptualization of oppositional defiant disorder: Implications for assessment and treatment. Clinical Child and Family Psychology Review, 2, 129-148. 
Harman, C., Rothbart, M. K. et Posner, M. I. (1997). Distress and attention interactions in early infancy. Motivation and Emotion, 21, 27-43.

Hoffmann, M. L. (2001). Toward a comprehensive empathy-based theory of prosocial moral development. Dans A. C. Bohart et D. J. Stipek (dir.), Constructive and destructive behaviour (p. 61-86). Washington, DC: American Psychological Association.

Hugues, C., Dunn, J. et White, A. (1998). Trick or treat?: Pattern of cognitive performance and executive function among "hard to manage" preschoolers. Journal of Child Psychology and Psychiatry, 39, 981-994.

Hugues, C., White, A., Sharpen, J. et Dunn, J. (2000). Antisocial, angry and unsympathetic: "Hard-to-manage" peer problems and possible cognitive influences. Journal of Child Psychology and Psychiatry, 41(2), 169-179.

Jolliffe, D. et Farrington, D. P. (2006). Development and validation of the Basic Empathy Scale. Journal of Adolescence, 29(4), 589-611.

Jurist, E. L. (2005). Mentalized affectivity. Psychoanalytic Psychology, 22 (3), 426-444.

Kozéki, B. et Berghammer, R. (1992). The role of empathy in the motivational structure of school children. Personality and Individual Differences, 13(2), 191-203.

Krebs, D. L. et Van Hesteren, F. (1994). The development of altruism: Toward an integrative model. Developmental Review, 14, 103-158.

Lafortune, D. (2008). Relation d'aide en criminologie. Document inédit, Université de Montréal, Canada.

Lanzetta, J. T. et Englis, B. G. (1989). Expectation of cooperation and competition and theirs effects on observers' vicarious emotional responses. Journal of Personality and Social Psychology, 56, 543-554.

Massé, L., Desbiens, N. et Lanaris, C. (2006). Les définitions cliniques des principales difficultés d'adaptation psychosociales à l'école. Dans L. Tousignant (dir.), Les troubles de comportements à l'école. Prévention, évaluation et intervention (pp. 2-4). Montréal: Gaëtan Morin.

Midgley, N. et Vrouva, I. (2012). Minding the child: Mentalization-based interventions with children, young people and their families. New York, NY: Routledge/Taylor et Francis Group.

Miller, P. A. et Jansen op de Haar, M. A. (1997). Emotional, cognitive, behavioral, and temperament characteristics of high-empathy children. Motivation and Emotion, 21(1), 109-125.

Ministère de l'Éducation, du Loisir et du Sport du Québec (2010). Document d'appui à la réflexion : rencontre sur l'intégration des élèves handicapés ou en difficulté. Repéré à http://www.mels.gouv.qc.ca/sections/publications/ index.asp?page=ficheetid $=1587$.

Mize, J. et Pettit, G. S. (2008). Social information processing and the development of conduct problems in children and adolescents: Looking beneath the surface. Dans C. Sharp, P. Fonagy et I. M. Goodyer (dir.), 
Social cognition and developmental psychopathology (p. 141-174). Oxford: Oxford University Press.

Penner, I. A., Dovidio, J. E., Piliavin, J. A. et Schroeder, D. A. (2005). Prosocial behaviour: Multilevel perspectives. Annual Review of Psychology, 56, 365-392.

Preston, S. D. et de Waal, F. B. M. (2002). Empathy: Its ultimate and proximate bases. Behavioral and Brain Sciences, 25(1), 1-20.

Sharp, C. (2006). Mentalizing problems in childhood disorders. Dans J. G. Allen et P. Fonagy (dir.), Handbook of mentalization-based treatment (p. 101-121). Chichester: John Wiley et Sons.

Sharp, C., Croudace, T. J. et Goodyer, I. M. (2007). Biased mentalizing in children aged seven to 11: Latent class confirmation of response styles to social scenarios and associations with psychopathology. Social Development, 16(1), 181-202.

Slade, A. (2006). Reflexive parenting program: Theory and development. Psychonalytic Inquiry, 26, 640-657.

Stetson, E. A. (1998). The development and validation of the empathic perspective taking scale (Thèse de doctorat inédite). Université de Denver, Colorado.

Strayer, J. et Roberts, W. (1989). Children's empathy and role taking: Child and parental factors, and relations to prosocial behavior. Journal of Applied Developmental Psychology, 10(2), 227-239.

Tardif, D., Joncas, B., Landry, L., Paquette, G., Leblanc, C., Gagnon, H., Bouffard, T. et Lafortune, L. (2003). Chantier sur la réussite des garçons, journée d'étude: un outil pour amorcer la réflexion. Document inédit, Québec, Canada.

Tremblay, R. et Royer, E. (1992). Lidentification des élèves qui présentent des troubles du comportement et l'évaluation de leurs besoins. Rapport présenté au ministère de l'Éducation, Direction de l'adaptation scolaire et des services complémentaires. Document inédit, Québec, Canada.

Twemlow, S., Fonagy, P. et Sacco, F. (2004). The role of the bystander in the social architecture of bullying and violence in schools and communities. Annals of New York Academy of Sciences, 1036, 215-232.

Twemlow, S. W. et Fonagy, P. (2005). A note on teachers who bully students in schools with differing levels of behavioral problems. American Journal of Psychiatry, 162 (12), 2387-2389.

Verheugt-Pleiter, A. J. E., Zevalkink, J. et Schmeets, M. G. J. (2008). Mentalizing in child therapy: Guidelines for clinical practitioners. London, England Karnac Books.

Vitaro, F. et Gagnon, C. (2008). Prévention des problèmes d'adaptation. Tome II: les problèmes externalisés. Québec: Presses de l'Université du Québec. 
Wellman, H. M. et Lagattuta, K. H. (2000). Developing understanding of mind. Dans S. Baron-Cohen, H. Tager-Flusberg et D. J. Cohen (dir.), Understanding other's minds: Perspectives from developmental cognitive neurosciences (p. 21-50). Oxford, England: Oxford University Press.

Wied, M., Goudena, P. P. et Matthys, W.. (2005). Empathy in boys with disruptive behavior disorders. Journal of Child Psychology and Psychiatry, 46(8), 867-880.

Winnicott, D. W. (1971). Playing and reality. New York: Basic Books.

\section{Correspondance :}

Émilie Girard

105, rue Bellerive

Saint-Lin-Laurentides, Québec

Canada J5M 0A4

Courriel: emilie.girard3@usherbrooke.ca

\section{Résumé}

La présente étude propose d'examiner les liens entre la capacité d'empathie, les comportements pro-sociaux, les biais de mentalisation et les troubles de comportement (TC) chez les garçons âgés de 7 à 12 ans. Pour ce faire, 51 garçons présentant divers niveaux de TC et leurs parents ont participé à la recherche. Les enfants ont rempli des questionnaires autoadministrés concernant l'empathie et les biais de mentalisation. Pour leur part, les parents ont rempli des questionnaires relatifs à l'empathie, aux comportements pro-sociaux et aux TC manifestés par leurs enfants. Les résultats démontrent que les biais de mentalisation négatifs et les comportements pro-sociaux prédisent $24,5 \%$ de la variance des TC. La capacité d'empathie quant à elle serait une variable médiatrice entre les comportements pro-sociaux et la présence des TC. Les résultats de la recherche permettront d'ajuster les programmes de prévention des TC et les interventions offertes aux enfants d'âge scolaire présentant ces troubles.

Mots-clés: empathie; biais de mentalisation; comportements prosociaux; troubles de comportement; enfants. 


\section{Abstract}

This study examines the relationship between empathy, pro-social behaviour, mentalization bias, and behavioural disorders (BD) in 7 to 12-year-old boys. Fifty-one boys, presenting different levels of BD, and their parents, participated in the research. Children completed self-administered measures on empathy and mentalization bias. The parents completed questionnaires on empathy, pro-social behaviour, and $\mathrm{BD}$ regarding their children. The results show that negative mentalization bias and pro-social behaviour predict $24.5 \%$ of the BD's variance. Empathy would be considered a mediating variable between pro-social behaviour and $\mathrm{BD}$. The results would be used to improve prevention programs and interventions offered to school-age children with BD.

Keywords: empathy; mentalization bias; pro-social behaviour; behavioural disorder; children. 